\title{
Review Article \\ Recent Developments in Nanoparticle-Based siRNA Delivery for Cancer Therapy
}

\author{
Jong-Min Lee, ${ }^{1}$ Tae-Jong Yoon, ${ }^{2}$ and Young-Seok Cho' \\ ${ }^{1}$ Department of Internal Medicine, Uijeongbu St. Mary's Hospital, The Catholic University of Korea College of Medicine, \\ Uijeongbu 480717, Republic of Korea \\ ${ }^{2}$ Department of Applied BioScience, CHA University, Sungnam 463836, Republic of Korea
}

Correspondence should be addressed to Young-Seok Cho; yscho@catholic.ac.kr

Received 31 December 2012; Accepted 30 May 2013

Academic Editor: John B. Vincent

Copyright (c) 2013 Jong-Min Lee et al. This is an open access article distributed under the Creative Commons Attribution License, which permits unrestricted use, distribution, and reproduction in any medium, provided the original work is properly cited.

\begin{abstract}
RNA interference (RNAi) is a gene regulation mechanism initiated by RNA molecules that enables sequence-specific gene silencing by promoting degradation of specific mRNAs. Molecular therapy using small interfering RNA (siRNA) has shown great therapeutic potential for diseases caused by abnormal gene overexpression or mutation. The major challenges to application of siRNA therapeutics include the stability and effective delivery of siRNA in vivo. Important progress in nanotechnology has led to the development of efficient siRNA delivery systems. In this review, the authors discuss recent advances in nanoparticle-mediated siRNA delivery and the application of siRNA in clinical trials for cancer therapy. This review will also offer perspectives on future applications of siRNA therapeutics.
\end{abstract}

\section{Introduction}

RNA interference (RNAi) is a process by which RNA molecules, with sequences complementary to a gene's coding sequence, induce degradation of corresponding messenger RNAs (mRNAs), thus blocking the translation of the mRNA into protein $[1,2]$. RNAi is initiated by exposing cells to long dsRNA via transfection or endogenous expression. dsRNAs are processed into smaller fragments (usually 21-23 nucleotides) of small interfering RNAs (siRNA) [3], which form a complex with the RNA-induced silencing complexes [4]. Introduction of siRNA into mammalian cells leads to downregulation of target genes without triggering interferon responses [3]. Molecular therapy using siRNA has shown great potential for diseases caused by abnormal gene overexpression or mutation, such as various cancers, viral infections, and genetic disorders, as well as for pain management. In the last 10 years, a tremendous effort has been made in biomedical therapeutic application of gene silencing in humans. Phase I studies of siRNA for the treatment of agerelated macular degeneration and respiratory syncytial virus provided promising data with no sign of nonspecific toxicity
$[5,6]$. However, there are many challenges to be overcome for siRNA cancer therapeutics, including safety, stability, and effective siRNA delivery.

The major barrier facing siRNA therapeutics is the efficiency of delivery to the desired cell type, tissue, or organ. siRNAs do not readily pass through the cell membrane due to their size and negative charge. Cationic liposomebased strategies are usually used for the cellular delivery of chemically synthesized or in vitro transcribed siRNA [7]. However, there are many problems with lipid-based delivery systems in vivo, such as rapid clearance by the liver and lack of target tissue specificity. Delivery systems can be categorized into physical methods, conjugation methods, and natural carrier (viruses and bacteria) and nonviral carrier methods [8]. DNA-based expression cassettes that express short hairpin RNA (shRNA) are usually delivered to target cells ex vivo by viruses and bacteria, and these modified cells are then reinfused back into the patient [9]. The popular adenovirus- and adeno-associated virus-derived vectors provide efficient delivery for shRNA expression [10]. However, there are problems with delivery using viral vectors, such as insertional mutagenesis and immunogenicity [11]. Nonviral 
gene delivery systems are highly attractive for gene therapy because they are safer and easier to produce than viral vectors. Nanotechnology has made significant advances in the development of efficient siRNA delivery systems. Current nonviral delivery systems can be categorized as organic and inorganic [12]. Organic complexes include lipid complexes, conjugated polymers, and cationic polymers, whereas inorganic nanoparticles include magnetic nanoparticles, quantum dots, carbon nanotubes, and gold nanoparticles. In this review, the authors discuss recent advances in nanoparticle-mediated siRNA delivery systems and the application of these systems in clinical trials for cancer therapy. Furthermore, we offer perspectives on future applications of siRNA therapeutics.

\section{Lipid-Based Nanovectors for Systemic siRNA Delivery}

2.1. Liposomes/Lipoplexes. Liposomes/lipoplexes have been extensively explored as nonviral vectors for plasmid and siRNA delivery [13]. Lipoplexes are complexes between cationic lipids and nucleic acids (mainly as plasmid DNA) [14]. Although neutral liposomes are more biocompatible than cationic lipids and have superior pharmacokinetics, they have low entrapment efficiency due to the lack of interaction between neutral lipids and anionic polynucleotides during formulation [15]. To increase entrapment efficiency, Landen Jr. et al. developed a method of formulating 1,2-dioleoylsn-glycero-3-phosphatidylcholine- (DOPC-) encapsulated siRNA liposomes that involves dissolving DOPC and siRNA in excess tertiary-butanol in the presence of the nonionic detergent Tween 20 [16]. DOPC-encapsulated siRNA targeting the oncoprotein EphA2 was highly effective in reducing EphA2 expression $48 \mathrm{~h}$ after administration of a single dose in an orthotopic model of ovarian carcinoma [16]. Treatment with DOPC-encapsulated siRNA via intravenous or intraperitoneal injections was highly effective in reducing both in vivo expression of target genes (e.g., EphA2, FAK, neuropilin-2, or IL-8) and tumor weight in mouse models of different human cancers [16-19]. In 2012, M.D. Anderson Cancer Center initiated a phase I dose-escalation trial for neutral liposome (DOPC) targeting of Eph2 in patients with advanced, recurrent cancer (http://www.clinicaltrials.gov/ct2/show/NCT01591356).

Cationic lipids, such as dioleoyl phosphatidylethanolamine and 1,2-dioleoyl-3-trimethylammonium-propane (DOTAP), form lipoplexes with negatively charged siRNA $[15,20]$. Cationic liposomes are routinely used for delivery of siRNA or plasmid DNA into mammalian cells in vitro [21]. However, surface interactions of cationic liposomes with the tumor cells produce an electrostatically derived binding site barrier effect, inhibiting further association of the delivery systems with tumor spheroids [22]. In addition, although cationic liposomes efficiently take up siRNA, limited success has been achieved with these systems in in vivo gene silencing, probably due to their intracellular stability and resultant failure to release siRNA contents [20]. Finally, the effectiveness of cationic liposomes has been limited by their toxicity. The use of cationic liposomes in vivo elicited dose-dependent toxicity and pulmonary inflammation by promoting release of reactive oxygen intermediates [23-25]. This effect was more pronounced with the multivalent cationic liposomes than with the monovalent cationic lipids, such as DOTAP [24].

The coating of liposomes with hydrophilic molecules, such as polyethylene glycol (PEG), reduced uptake by the reticuloendothelial system (RES), resulting in enhanced circulatory half-life [26]. In 2006, Santel et al. developed a novel liposomal siRNA formulation based on cationic lipids (siRNA-lipoplex/AtuPLEX), containing neutral fusogenic and PEG-modified lipid components, for improved pharmacokinetics and cellular uptake, and more efficient siRNA release $[27,28]$. Using this formulation to target endothelia-specific genes, such as CD31 (platelet endothelial cell adhesion molecule-1) or TIE-2, they demonstrated downregulation of the corresponding mRNAs and proteins in mice [28]. Atu027 is a lipoplexed siRNA molecule specifically targeting the expression of protein kinase $\mathrm{N} 3$, which has been identified as a downstream effector of the phosphoinositol-3-kinase signaling pathway [29]. Atu027 has been reported to inhibit lymph node metastasis in orthotopic prostate and pancreatic cancer mouse models and to inhibit hematogenous metastasis to the target organ lung in various mouse lung metastasis models [29, 30]. Silence Therapeutics (London, UK) is performing a phase I trial of Atu027, which was well tolerated up to a dose of $0.180 \mathrm{mg} / \mathrm{kg}$ and was not associated with dose-dependent toxicities, in patients with colorectal cancer metastasized to the liver [31]. Dose escalation is currently being investigated. Using liposomal encapsulation of siRNA nanoparticles, another delivery platform, tauRNAi, has been developed by Marina Biotech (Bothell, WA, USA). This drug is in the preclinical stage for hepatocellular carcinoma [32].

2.2. Stable Nucleic Acid Lipid Particles and Lipidoids. Solid lipid-based systems have been developed as alternatives to emulsions, liposomes, microparticles, and polymeric nanoparticles for systemic delivery of siRNA and include stable nucleic acid lipid particles (SNALPs) and cationic solid-lipid nanoparticles [33, 34]. Jeffs et al. developed a new "spontaneous vesicle formation" method for the preparation of rapid and reproducible stabilized plasmid lipid particles for nonviral, systemic gene therapy [35]. Using this controlled, stepwise dilution method, Morrissey et al. developed SNALPs, which are PEG-conjugated lipid nanoparticles comprised of siRNA encapsulated inside a lipid bilayer of neutral lipids and PEG-lipid fusion regulators [33]. Stabilized siRNA targeting hepatitis B virus (HBV) RNA was incorporated into SNALPs and administered by intravenous injection into mice carrying replicating $\mathrm{HBV}$, resulting in reduction of the level of HBV DNA. Furthermore, reductions were seen in serum HBV DNA for up to 6 weeks with weekly dosing. Zimmermann et al. have demonstrated that intravenous injection of ApoB-targeting siRNAs encapsulated in SNALPs resulted in significant dose-dependent silencing $A p o B$ mRNA in the livers of both mice and nonhuman primates [36]. A single administration of $2.5 \mathrm{mg} / \mathrm{kg}$ SNALP-formulated siRNA was well tolerated and reduced $A p o B$ mRNA expression 
in the liver by up to $90 \%$, lasting for 11 days at the highest siRNA dose. SNALP-formulated siRNA targeting the essential cell-cycle proteins polo-like kinase 1 (PLK1) and kinesin spindle protein (KSP) showed potent antitumor efficacy in both hepatic and subcutaneous tumor models [37]. Tekmira Pharmaceuticals Corporation (Burnaby, BC, Canada) initiated a phase I trial of SNALP-encapsulated siRNA targeting PLK1 (TKM 080301) in December 2010 (http://www.clinicaltrials.gov/ct2/show/NCT01262235). This is dose-escalation trial conducted at multiple clinical centers, designed to determine TKM 080301 safety, tolerability, and pharmacokinetics in adult patients with solid tumors or lymphomas that are refractory to standard therapy or for whom there is no standard therapy. Alnylam Pharmaceuticals (Cambridge, MA, USA) has developed SNALP-formulated siRNAs targeting vascular endothelial growth factor (VEGF) and KSP in ALN-VSP02, the first dual-targeted siRNA drug. In April 2009, a phase I dose-escalation trial was initiated (http://www.clinicaltrials.gov/ct2/show/NCT00882180). Interim data from pharmacodynamic measurements provided preliminary evidence of clinical activity for the treatment of advanced solid tumors with liver involvement. Additional results from the initial 28 patients in the first six-dose cohorts demonstrated that ALN-VSP02 was generally well tolerated at the highest dose $(1.25 \mathrm{mg} / \mathrm{kg})$ [38]. The study has not yet reached a maximum tolerated dose and the trial continues to enroll patients in a dose-escalating manner. In another phase I trial, several patients with stable disease have advanced to a multicenter, open label, extension study to collect long-term safety data (http://www.clinicaltrials.gov/ct2/show/NCT01158079).

Lipidoid nanoparticles are lipid-like delivery molecules comprised of cholesterol and PEG-modified lipids specific for delivery of specific siRNA [38]. To improve SNALP-mediated delivery, Akinc et al. developed a new chemical method to allow rapid synthesis of a large library of lipidoids and tested their efficacy in siRNA delivery [39]. The leading candidate, the $98 \mathrm{~N}_{12}-5$ lipidoid-based siRNA formulation, showed $75 \%-90 \%$ reduction in ApoB or FVII factor expression in hepatocytes in nonhuman primates and mice $[39,40]$. This formulation facilitated gene silencing at orders-of-magnitude lower doses of siRNA than those required by the original SNALP formulation, resulting in reduced toxicity [41].

\section{Polymeric Nanoparticles}

Polymeric nanoparticles are solid, biodegradable, colloidal systems that have been widely investigated as drug or gene carriers [42]. Polymeric nanoparticles are classified into two major categories, natural polymers and synthetic polymers. Natural polymers for siRNA delivery include cyclodextrin, chitosan, and atelocollagen [12]. Of the synthetic polymers, polyethyleneimine (PEI), poly(dl-lactide-co-glycolide) (PLGA), and dendrimers have been intensively investigated [8].

3.1. Cyclodextrin Nanoparticle. Cyclodextrins are natural polymers generated during the bacterial digestion of cellulose and can form water-soluble inclusion complexes with small molecules and portions of large compounds [43]. Hu-Lieskoven et al. developed the cyclodextrin-containing polycation system for the targeted delivery of siRNA [44]. This system consists of a cyclodextrin-containing polymer, PEG for stability, and human transferrin as the targeting ligand for binding to transferrin receptors, which are often overexpressed on cancer cells. This targeted nanoparticle system, called CALLA-01, was developed for the first siRNA phase I trial by Calando Pharmaceuticals (Pasadena, CA, USA) [45]. The siRNA in CALLA-01 is designed to inhibit tumor growth via a mechanism to reduce expression of the M2 subunit of ribonucleotide reductase (R2). Patients with solid cancers refractory to standard-of-care therapies were administered targeted nanoparticles via IV infusion on days $1,3,8$, and 10 of a 21-day cycle [45]. Successful delivery of targeted nanoparticles was confirmed by the presence of intracellular nanoparticles in tumor biopsies from melanoma patients after treatment. Furthermore, knockdown of the M2 subunit of R2 was confirmed in tumor biopsies from these patients by quantitative reverse transcription-polymerase chain reaction (qRT-PCR) and by immunohistochemical staining in the patients treated with the highest dosage. This study demonstrated that siRNA administered systemically to humans may result in specific gene inhibition by an RNAimediated mechanism of action.

3.2. Chitosan Nanoparticles. Chitosan, a type of naturally occurring polysaccharide, has been extensively studied for the delivery of plasmid DNA and siRNA in vitro and in vivo [46-48]. The advantages of chitosan include mucoadhesivity, biocompatibility, biodegradability, and low cost of production. However, results of studies of siRNA delivery have been inconsistent due to discrepancies between experiments [46, 49]. In addition, high molecular weight chitosans are cytotoxic, thus limiting their use in clinical trials [50]. They still also lack the buffering capacity needed for endosomolysis, which is essential to siRNA release from the endosome [51].

3.3. Polyethyleneimine. PEI, a commonly used cationic polymeric drug carrier with high transfection efficiency, has been widely investigated for siRNA delivery $[8,12,51]$. PEI forms small and compact structures, spontaneously forming polyplexes, with negatively charged siRNA through a simple and short polycation process [12]. The PEI-siRNA complexes protect siRNA from nuclease degradation, resulting in prolonged half-life. In addition, complete encapsulation of siRNA prevents avoid off-target effects such as immune activation by a toll-like receptor dependent mechanism [52]. However, PEI complexes have been associated with significant toxicity issues limiting their broad use in clinical trials [50]. Molecular mechanisms of PEI cytotoxicity include membrane damage and activation of a mitochondriamediated apoptotic program due to PEI-induced channel formation in the outer mitochondrial membrane $[53,54]$.

3.4. PLGA. PLGA is a copolymer of glycolic acid and lactic acid and a US Food and Drug Administrationapproved biodegradable polymer [55]. PLGA has been used 
as a nanocarrier for plasmid DNA and siRNA delivery in recent years. The advantages of PLGA-based siRNA delivery include high stability, facile cellular uptake by endocytosis, ability to target specific tissues or organs by adsorption or ligand binding, biodegradability, low toxicity, and sustained release characteristics [56]. However, PLGA could not be applied efficiently in siRNA delivery due to the lower electrostatic interaction between PLGA and siRNA and less efficient endosomal escape and release of siRNA $[8,56]$. To overcome these problems, the surface of PLGA can be decorated with various cationic nanoparticles such as DOTAP, PEI, or polyamine [51].

3.5. Dendrimers. Dendrimers are synthetic, highly branched monodisperse, and usually highly symmetric, spherical macromolecules with three-dimensional nanometric structure. The unique structural features such as tunable structure and molecular size, large number of accessible terminal functional groups, and ability to encapsulate cargos add to their potential as drug carriers [57]. Polycationic dendrimers such as poly(amidoamine) (PAMAM) and poly(propylenimine) (PPI) dendrimers have been studied for siRNA delivery in recent years. PAMAM dendrimers have become the most used dendrimer-based carriers for gene delivery because of the ease of synthesis and commercial availability. However, PAMAMs were demonstrated to be cytotoxic, predominately related to apoptosis mediated by mitochondrial dysfunction [58]. Cytotoxicity can be reduced by various modifications without compromising gene silencing. Surface-modified and cationic PAMAM dendrimers show very low cytotoxicity, even at high concentrations and efficiently penetrated cancer cells in vitro [59]. PPI dendrimers were also used to formulate siRNA nanoparticles, and these nanoparticles showed efficient gene silencing [60]. Dendrimer-conjugated magnetofluorescent nanoworms (dendriworms) were developed to achieve siRNA delivery in a transgenic murine model of glioblastoma [61]. These siRNA-carrying dendriworms maximized endosomal escape to robustly produce protein target knockdown and were tolerated well in mouse brain.

\section{Inorganic Nanoparticles}

A number of inorganic nanoparticles have been emerging as potential siRNA delivery systems devised for simultaneous imaging and therapeutic purposes. They include carbon nanotubes (CNTs) and metals such as iron oxide, quantum dots (QDs), and gold. CNTs are nanomaterials, with interesting physical and chemical properties, and have recently emerged as a new option for cancer treatment, bioengineering, and gene therapy. It has been proposed that CNTs easily cross the plasma membrane and translocate directly into cytoplasm of target cells due to their nanoneedle structure, using an endocytosis-independent mechanism without inducing cell death $[62,63]$. CNTs are classified as single-walled CNTs and multiwalled CNTs [64]. Several functionalized CNTs have been designed and tested for the purpose of siRNA delivery. Zhang et al. used single-walled CNTs functionalized with $-\mathrm{CONH}-\left(\mathrm{CH}_{2}\right)_{6}-\mathrm{NH}_{3}{ }^{+} \mathrm{Cl}^{-}$as siRNA carriers [65]. They released the siRNA from the nanotube side-wall to silence telomerase reverse transcriptase expression, which considerably suppressed tumor growth. CNTs functionalized with amine-terminated PEG (phospholipid (PL)-PEG2000$\mathrm{NH}_{2}$ ) were shown to be efficient in siRNA delivery into human T cells [66]. Ammonium-functionalized CNTs and dendron-CNTs have also been reported to be efficient in siRNA delivery with low cytotoxicity $[67,68]$. A comparative study of antitumor activity of the proprietary cytotoxic siRNA sequence (siTOX) delivered either by cationic liposomes (DOTAP : cholesterol) or amino-functionalized MWNT- $\mathrm{NH}^{+3}$ in a human lung xenograft model demonstrated that only MWNT-NH ${ }^{+3}$ : siRNA complexes administered intratumorally could elicit delayed tumor growth and increased survival of xenograft-bearing animals [69]. However, several studies have discussed the potential toxicity of CNTs although the underlying mechanisms are uncertain [70, 71].

Magnetic nanoparticles, including superparamagnetic iron oxide nanoparticles (SPIOs) and magnetic iron tetroxide particles, emerged as feasible nanotheranostics for tumor imaging and drug delivery due to their distinct characteristics [72]. The large surface area of SPIOs makes their functional modification feasible, enabling the conjugation of targeting molecules, drugs, and imaging agents [73]. Moore and her colleagues reported the synthesis and characterization of a new dual-purpose probe for the simultaneous noninvasive imaging and delivery of siRNAs to tumors [74]. This probe consists of magnetic nanoparticles (SPIOs for magnetic resonance imaging) conjugated with Cy5.5 dye (for near-infrared fluorescence imaging (NIRF)) and myristoylated polyarginine peptide for membrane translocation. A nanoparticle probe (MN-NIRF-siSurvivin) targeting the antiapoptotic gene Birc5, which encodes survivin, significantly increased cancer cell apoptosis and necrosis in vitro and in xenograft mouse models. Therefore, use of MN-NIRFsiSurvivin conjugates combining siRNA delivery with a dualimaging modality (magnetic resonance imaging and NIRF) was feasible for multimodality imaging and targeted gene delivery. Lee et al. developed manganese-doped magnetismengineered iron oxide (MnMEIO) nanoparticles conjugated to a cancer-specific targeting moiety the Arg-Gly-Asp (RGD) peptide, which specifically binds to tumors expressing $\alpha_{\mathrm{v}} \beta_{3}$ integrin, and Cy5-dye-labeled siGFP, which inhibits the expression of green fluorescence protein (GFP) [75]. The constructed nanoparticle (MnMEIO-siGFP-Cy5/PEG-RGD) showed specific internalization and target gene silencing in $\alpha_{\mathrm{v}} \beta_{3}$ integrin-expressing breast cancer MDA-MB-435 cells. An additional advantage of iron oxide nanoparticle delivery systems is that they can be delivered in a targeted manner to a desired region by applying an external magnetic field [76].

Semiconductor QDs, which are light-emitting nanoparticles, have been increasingly used as biological imaging and labeling probes [77]. QDs also have the potential of serving as photostable beacons for siRNA delivery and imaging [78-80]. However, the major problem in using QDs as multifunctional imaging probes and delivery systems is their toxicity because most well-established QDs are composed of highly toxic elements, such as cadmium, selenium, or tellurium [81]. 
Recently, nontoxic QDs, which were developed by a novel sonochemical approach for the high-throughput synthesis of a library of biocompatible ZnS-AgInS2 QDs, showed great potential for imaging and siRNA delivery in vitro with negligible cytotoxicity [82]. However, a more thorough investigation of their long-term cytotoxicity is necessary before they can be used in vivo.

Gold nanoparticles (AuNPs) have emerged as a promising siRNA delivery carrier due to their excellent biocompatibility, ease of synthesis, high surface-to-volume ratio, and facile surface functionalization [83]. Recently, various types of AuNPs have been widely investigated for siRNA delivery. These include AuNPs functionalized with cationic quaternary ammonium or branched PEI, cationic lipid bilayer coated AuNPs, and oligonucleotide-modified AuNPs [83-85]. Gold nanorods also have the potential to deliver siRNA to target cells or tissues. The Prasad group developed gold nanorodDARPP-32 siRNA complexes to target and reduce expression of the key proteins (DARPP-32, extracellular signal-regulated kinase (ERK), and protein phosphatase 1 (PP-1)) in the dopaminergic signaling pathway in the brain for therapy of drug addiction [86]. Using dark-field imaging and confocal microscopy, they demonstrated that the siRNA was efficiently delivered into dopaminergic neuronal (DAN) cells after treatment with the gold nanorod-siRNA conjugates. Moreover, the delivery of nanoplexes containing siRNA targeted to the DARPP-32 gene in DAN cells resulted in the silencing not only of DARPP-32, but also of other key downstream effector molecules in this pathway, such as ERK and PP-1, with greater efficiency than commercial transfection agents. Recently, Kim et al. reported that AuNPs stably functionalized with covalently attached oligonucleotides activate immune-related genes and pathways in human peripheral blood mononuclear cells, but not an immortalized, lineage-restricted cell line [87]. These later findings suggest that assessment of the toxic potential of engineered nanoparticles in immortalized, lineage-restricted cell lines may not predict their phenotypic effects in relevant biological systems.

\section{Targeted Delivery}

Significant advances have been made in the development of efficient siRNA delivery in nonviral vector systems, such as cationic lipids and polymers. However, a major problem with these approaches is that a large amount of siRNA has to be administered for efficient gene silencing. Moreover, cell type-specific targeting can prevent off-target effects, thus reducing the side effects of the therapeutics. A common approach for targeted delivery of siRNA to specific cells or tissues is conjugation to ligands such as antibodies, aptamers, and peptides which specifically bind to the corresponding moieties on target cells. Song and colleagues developed a protamine-antibody fusion protein for systemic and targeted siRNA delivery [88]. They fused protamine, a protein that binds nucleic acids, to a Fab directed against the human immunodeficiency virus type 1 (HIV-1) envelope protein and mixed the siRNA with the fusion protein. Treatment with the fusion protein mixed with siRNA targeted to the HIV-1 gag protein suppressed viral replication in infected primary
$\mathrm{T}$ cells. Kumar et al. demonstrated $\mathrm{T}$ cell-specific siRNA delivery in a preclinical animal model [89]. In this study, a CD7-specific single-chain antibody was conjugated to oligo-

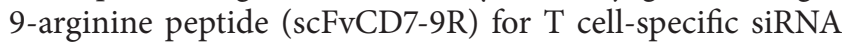
delivery in humanized mice. Antiviral siRNAs complexed to scFvCD7-9R were shown to be delivered to naïve T cells and suppressed HIV replication in HIV-infected mice.

Nucleic-acid aptamers, which are normally selected from a large random-sequence pool to bind to a specific target molecule, have been explored for targeted siRNA delivery as an alternative to antibodies. Aptamers have advantages, such as high selective binding to proteins and receptors, ready-to-use chemical synthesis, process-compatible storability, and low immunogenicity [12]. McNamara II et al. have developed aptamer-siRNA chimeric RNAs for targeted delivery of siRNA [90]. The aptamer portions of the chimeras were introduced for specific binding to prostate-specific membrane antigen (PSMA), a cell-surface receptor overexpressed in prostate cancer cells and tumor endothelium, whereas the siRNA portion targeted the expression of survival genes. The chimeric RNA was demonstrated to bind only PSMA-expressing cells, resulting in depletion of siRNA target proteins and cell death. In addition, treatment with the chimeric RNA specifically inhibited tumor growth and mediated tumor regression in a xenograft model of prostate cancer. The aptamer-siRNA chimera is a promising targeted approach for siRNA delivery because RNA is not recognized by antibodies. However, more RNA and DNA aptamers must be developed for specific cancer or disease markers to expand the use of aptamer delivery approach. Recently, extensive studies have been performed to develop an RNA nanoparticle-based siRNA vector [91]. Packaging RNA is a 117-nt RNA molecule that constitutes one of the six packaging RNA subunits of the phi29 bacteriophage DNA packaging motor [92]. Chemically modified and folate receptor-targeted packaging RNA nanoparticles for siRNA delivery showed high in vivo stability with a blood half-life of 5 to $10 \mathrm{~h}$ and were retained in cancer tissue for more than $8 \mathrm{~h}$. Tumortargeted delivery and efficacy of gene silencing have also been in xenograft tumor models [92-94].

Another strategy for enhanced delivery of siRNA involves covalent conjugates to cell penetrating peptides (CPPs) or protein transduction domains [95]. The cationic nature of CPPs is crucial for their ability to bind and pass through the anionic cellular membrane. CPP conjugates of siRNA exhibited gene-silencing effects on target receptor proteins in various mammalian cell lines. However, conjugation of cationic peptides to anionic siRNA may neutralize and reduce the penetrating efficacy of these peptides [95]. In addition, CPP-siRNA conjugates may exhibit cytotoxicity caused by cell membrane perturbation or immunogenicity [96].

Recently, we have developed a new approach for targeted delivery and expression of siRNAs in vivo using DNAbased siRNA expression nanocassettes and receptor-targeted nanoparticles [97]. This new nanoparticle consists of an amphiphilic polymer-coated QD conjugated to 10 to 20 DNA nanocassettes that contain a U6 promoter and shRNA gene for in vivo siRNA gene expression following delivery to target cells. The nanoparticle was conjugated to the amino terminal 
TABLE 1: siRNA cancer therapeutics in clinical trials.

\begin{tabular}{|c|c|c|c|c|c|c|c|}
\hline Drug & Company & Vehicle & Target & Disease & $\begin{array}{l}\text { Delivery } \\
\text { route }\end{array}$ & Phase & Stage \\
\hline CALAA-01 & $\begin{array}{l}\text { Calando } \\
\text { Pharma }\end{array}$ & $\begin{array}{c}\text { Cyclodextrin } \\
\text { nanoparticle, } \\
\text { Transferrin, PEG }\end{array}$ & $\begin{array}{l}\text { M2 subunit of } \\
\text { ribonucleotide } \\
\text { reductase }\end{array}$ & Solid tumors & IV & I & $\begin{array}{l}\text { Ongoing, } \\
\text { not } \\
\text { recruiting }\end{array}$ \\
\hline Atu027 & $\begin{array}{c}\text { Silence } \\
\text { Therapeutics }\end{array}$ & $\begin{array}{c}\text { Liposomes } \\
\text { (Lipoplexes, Cationic } \\
\text { lipid) }\end{array}$ & Protein kinase N3 & Solid tumors & IV & I & Completed \\
\hline \multirow[t]{2}{*}{ ALN-VSP02 } & $\begin{array}{l}\text { Alnylam } \\
\text { Pharma }\end{array}$ & SNALP & VEGF and KSP & $\begin{array}{l}\text { Solid tumors with } \\
\text { liver involvement }\end{array}$ & IV & I & Completed \\
\hline & & & & Solid tumors & IV & I & Completed \\
\hline \multirow[t]{2}{*}{ TKM 080301} & $\begin{array}{l}\text { Tekmira } \\
\text { Pharma }\end{array}$ & SNALP & Polo-kinase-1 & Solid tumors & IV & I & Recruiting \\
\hline & & & & $\begin{array}{l}\text { Solid tumors with } \\
\text { liver involvement }\end{array}$ & IV & I & Completed \\
\hline siRNA-EphA2-DOPC & $\begin{array}{c}\text { M.D. } \\
\text { Anderson } \\
\text { Cancer } \\
\text { Center }\end{array}$ & $\begin{array}{l}\text { Liposomes (neutral } \\
\text { liposomes) }\end{array}$ & EphA2 & Solid tumors & IV & I & $\begin{array}{l}\text { Not yet } \\
\text { open }\end{array}$ \\
\hline \multirow[t]{2}{*}{ siG12D LODER } & Silenseed Ltd & $\begin{array}{l}\text { Polymer matrix } \\
\text { (LODER polymer) }\end{array}$ & KRASG12D & $\begin{array}{l}\text { Pancreatic ductal } \\
\text { adenocarcinoma }\end{array}$ & $\begin{array}{l}\text { EUS biopsy } \\
\text { needle }\end{array}$ & I & $\begin{array}{l}\text { Ongoing, } \\
\text { recruiting }\end{array}$ \\
\hline & & & & & & II & $\begin{array}{c}\text { Not yet } \\
\text { open }\end{array}$ \\
\hline
\end{tabular}

fragment of urokinase plasminogen activator (uPA), which targets its cellular receptor, uPAR. This receptor is highly expressed in tumor, angiogenic endothelial, and stromal cells in many types of human cancers $[98,99]$. Targeted delivery and gene-silencing efficiency of firefly luciferase siRNA nanogenerators were demonstrated in tumor cells and in animal tumor models. Moreover, delivery of survivin siRNAexpressing nanocassettes into tumor cells induced apoptotic cell death and sensitized cells to chemotherapeutic drugs. In cultured cells, the extent of targeted gene knockdown by survivin siRNA-expressing DNA nanocassettes using the uPAR-targeted nanoparticle delivery system was similar to that achieved with SV40-nuclear localization signal- (NLS-) mediated internalization of the QD-survivin siRNA nanocassettes. However, SV40-NLS-QD-siRNA nanocassettes could not be used for in vivo delivery due to their lack of specificity. These findings suggest that a receptor-targeted nanoparticle carrier allows efficient delivery into target tissues as well as intracellular delivery.

\section{Clinical Trials}

Currently, there are six cancer clinical trials underway using nanoparticle-based siRNA delivery, all in Phase I, evaluating the initial safety and utility of these treatments (Table 1). All the nanoparticle-formulated siRNA delivery systems for cancer therapy that are currently in clinical trials are based on polymers or liposomes. Since CALLA-01 was developed for the first siRNA phase I trial by Calando Pharmaceuticals, several other companies, including Tekmira, Alnylam, Silence Therapeutics, Marina, and others, have introduced siRNA nanoparticle products in either the preclinical or clinical phases. Silenseed Ltd (Jerusalem, Israel) initiated a phase I dose-escalation trial for siG12D LODER local drug eluter (LODER) (http://www.clinicaltrials .gov/ct2/show/NCT01188785). The siG12D LODER is a miniature biodegradable polymeric matrix that encompasses siRNA target to KRASG12D mRNA (siG12D) drug, designed to release the drug locally within a pancreatic tumor, for a prolonged period of 8 weeks. The siG12D LODER is injected into the patient's tumor with needle during an endoscopic ultrasound (EUS) biopsy procedure. The majority of pancreatic ductal adenocarcinomas involves mutations in the KRAS oncogene with the most common being G12D; therefore, administration of KRASG12D siRNA has the potential to silence KRAS, leading to apoptosis of the cancer cells and, thereby, slowing and halting tumor growth. In an upcoming Phase II study, a single dose of 3,000 $\mu \mathrm{g}$ (eight 375- $\mu \mathrm{g}$ siG12D LODERs) will be administered to patients with unresectable, locally advanced pancreatic cancer, in combination with chemotherapy treatment (http://www.clinicaltrials.gov/ct2/show/NCT01676259).

\section{Conclusions and Future Perspectives}

Since RNAi was discovered, various nonviral vector delivery systems for siRNA delivery have been explored extensively. Although significant advances have been made in the development of efficient in vivo siRNA delivery, there are still many challenges and barriers that must be overcome to achieve the ideal formulation in terms of selectivity, efficacy, and safety. Only a few nanoparticle-based siRNA delivery systems have been approved by the FDA and are in clinical trials for cancer therapy. Delivery systems can improve specificity of cancer 
cell targeting, prevent non-specific delivery of siRNA, and may also protect the siRNA during transport. Nanoparticles conjugated to the targeting ligand for effective siRNA delivery increase the chance of binding the tumor surface receptor; however, the process also increases the overall size of the nanoparticle. The PEG coating of nanoparticles reduces uptake by RES, resulting in enhanced circulatory half-life, but reduces targeting specificity because PEG molecules sterically disrupt selective conjugation. Thus, the selection of appropriate cell-specific targeting moieties and careful design of stable and potent nanoparticle delivery systems is required for future development.

Other major challenges for RNAi-based cancer therapeutics include controlling the specificity of the siRNA, minimizing off-target effects, increasing resistance to nuclease degradation, and avoiding immune responses such as $\alpha / \beta$ interferons, RNA-dependent kinase effects, and toll-like immunity. Chemical modification of the siRNA, such as inserting a $2^{\prime}$-O-methyl ribose in the nucleotide in the second position of the guide strand, could reduce silencing of most off-target transcripts with complementarity to the siRNA guide [100]. Dual-targeted siRNA drugs, such as ALN-VSP02, which targets VEGF and KSP, may reduce the potential for off-target gene silencing and increase the chances of knocking down the desired target.

Various nanoparticle-based delivery systems such as cationic lipids, polymers, dendrimers, and inorganic nanoparticles have been demonstrated to provide effective and efficient siRNA delivery in vitro and in vivo. Future studies must focus on the in vivo safety profiles of the various delivery systems, including undesirable immune stimulation and cytotoxicity. It is critical to develop safe, biocompatible, and biodegradable nanoparticle delivery systems for the clinical application of RNAi-based cancer therapeutics.

\section{Authors' Contribution}

J.-M. Lee and T.-J. Yoon contributed equally to this work.

\section{Acknowledgments}

This work was supported by National Research Foundation of Korea (NRF) Grant funded by the Ministry of Education, Science, and Technology (2012-0006902).

\section{References}

[1] G. J. Hannon, "RNA interference," Nature, vol. 418, no. 6894, pp. 244-251, 2002.

[2] P. A. Sharp, "RNAi and double-strand RNA," Genes \& Development, vol. 13, no. 2, pp. 139-141, 1999.

[3] S. M. Elbashir, J. Harborth, W. Lendeckel, A. Yalcin, K. Weber, and T. Tuschl, "Duplexes of 21-nucleotide RNAs mediate RNA interference in cultured mammalian cells," Nature, vol. 411, no. 6836, pp. 494-498, 2001.

[4] E. Bernstein, A. A. Caudy, S. M. Hammond, and G. J. Hannon, "Role for a bidentate ribonuclease in the initiation step of RNA interference," Nature, vol. 409, no. 6818, pp. 363-366, 2001.
[5] E. Check, "A crucial test," Nature Medicine, vol. 11, no. 3, pp. $243-$ 244, 2005.

[6] J. DeVincenzo, J. E. Cehelsky, R. Alvarez et al., "Evaluation of the safety, tolerability and pharmacokinetics of ALN-RSV01, a novel RNAi antiviral therapeutic directed against respiratory syncytial virus (RSV)," Antiviral Research, vol. 77, no. 3, pp. 225231, 2008.

[7] L. Aagaard and J. J. Rossi, "RNAi therapeutics: principles, prospects and challenges," Advanced Drug Delivery Reviews, vol. 59, no. 2-3, pp. 75-86, 2007.

[8] X. Yuan, S. Naguib, and Z. Wu, "Recent advances of siRNA delivery by nanoparticles," Expert Opinion on Drug Delivery, vol. 8, no. 4, pp. 521-536, 2011.

[9] J. C. Burnett, J. J. Rossi, and K. Tiemann, "Current progress of siRNA/shRNA therapeutics in clinical trials," Biotechnology Journal, vol. 6, no. 9, pp. 1130-1146, 2011.

[10] Y. P. Liu and B. Berkhout, "MiRNA cassettes in viral vectors: problems and solutions," Biochimica et Biophysica Acta, vol. 1809, no. 11-12, pp. 732-745, 2011.

[11] P. L. Sinn, S. L. Sauter, and P. B. McCray Jr., "Gene therapy progress and prospects: development of improved lentiviral and retroviral vectors-design, biosafety, and production," Gene Therapy, vol. 12, no. 14, pp. 1089-1098, 2005.

[12] Y. Wang, Z. Li, Y. Han, L. H. Liang, and A. Ji, "Nanoparticlebased delivery system for application of siRNA in vivo," Current Drug Metabolism, vol. 11, no. 2, pp. 182-196, 2010.

[13] D. B. Fenske and P. R. Cullis, "Liposomal nanomedicines," Expert Opinion on Drug Delivery, vol. 5, no. 1, pp. 25-44, 2008.

[14] A. Elouahabi and J.-M. Ruysschaert, "Formation and intracellular trafficking of lipoplexes and polyplexes," Molecular Therapy, vol. 11, no. 3, pp. 336-347, 2005.

[15] S. Y. Wu and N. A. J. McMillan, "Lipidic systems for in vivo siRNA delivery," AAPS Journal, vol. 11, no. 4, pp. 639-652, 2009.

[16] C. N. Landen Jr., A. Chavez-Reyes, C. Bucana et al., “Therapeutic EphA2 gene targeting in vivo using neutral liposomal small interfering RNA delivery," Cancer Research, vol. 65, no. 15, pp. 6910-6918, 2005.

[17] J. Halder, A. A. Kamat, C. N. Landen Jr. et al., "Focal adhesion kinasetargeting using in vivo short interfering RNA delivery in neutral liposomes for ovarian carcinoma therapy," Clinical Cancer Research, vol. 12, no. 16, pp. 4916-4924, 2006.

[18] M. J. Gray, G. Van Buren, N. A. Dallas et al., "Therapeutic targeting of neuropilin-2 on colorectal carcinoma cells implanted in the murine liver," Journal of the National Cancer Institute, vol. 100, no. 2, pp. 109-120, 2008.

[19] W. M. Merritt, Y. G. Lin, W. A. Spannuth et al., "Effect of interleukin-8 gene silencing with liposome-encapsulated small interfering RNA on ovarian cancer cell growth," Journal of the National Cancer Institute, vol. 100, no. 5, pp. 359-372, 2008.

[20] B. Ozpolat, A. K. Sood, and G. Lopez-Berestein, "Nanomedicine based approaches for the delivery of siRNA in cancer," Journal of Internal Medicine, vol. 267, no. 1, pp. 44-53, 2010.

[21] S. Taetz, A. Bochot, C. Surace et al., "Hyaluronic acid-modified DOTAP/DOPE liposomes for the targeted delivery of antitelomerase siRNA to CD44-expressing lung cancer cells," Oligonucleotides, vol. 19, no. 2, pp. 103-116, 2009.

[22] K. Kostarelos, D. Emfietzoglou, A. Papakostas, W.-H. Yang, Å. Ballangrud, and G. Sgouros, "Binding and interstitial penetration of liposomes within avascular tumor spheroids," International Journal of Cancer, vol. 112, no. 4, pp. 713-721, 2004. 
[23] S. Dokka, D. Toledo, X. Shi, V. Castranova, and Y. Rojanasakul, "Oxygen radical-mediated pulmonary toxicity induced by some cationic liposomes," Pharmaceutical Research, vol. 17, no. 5, pp. 521-525, 2000.

[24] S. Spagnou, A. D. Miller, and M. Keller, "Lipidic carriers of siRNA: differences in the formulation, cellular uptake, and delivery with plasmid DNA," Biochemistry, vol. 43, no. 42, pp. 13348-13356, 2004.

[25] H. Lv, S. Zhang, B. Wang, S. Cui, and J. Yan, "Toxicity of cationic lipids and cationic polymers in gene delivery," Journal of Controlled Release, vol. 114, no. 1, pp. 100-109, 2006.

[26] M. Uner and G. Yener, "Importance of solid lipid nanoparticles (SLN) in various administration routes and future perspectives," International Journal of Nanomedicine, vol. 2, no. 3, pp. 289-300, 2007.

[27] A. Santel, M. Aleku, O. Keil et al., "RNA interference in the mouse vascular endothelium by systemic administration of siRNA-lipoplexes for cancer therapy," Gene Therapy, vol. 13, no. 18, pp. 1360-1370, 2006.

[28] A. Santel, M. Aleku, O. Keil et al., "A novel siRNA-lipoplex technology for RNA interference in the mouse vascular endothelium," Gene Therapy, vol. 13, no. 16, pp. 1222-1234, 2006.

[29] M. Aleku, P. Schulz, O. Keil et al., "Atu027, a liposomal small interfering RNA formulation targeting protein kinase N3, inhibits cancer progression," Cancer Research, vol. 68, no. 23, pp. 9788-9798, 2008.

[30] A. Santel, M. Aleku, N. Röder et al., "Atu027 prevents pulmonary metastasis in experimental and spontaneous mouse metastasis models," Clinical Cancer Research, vol. 16, no. 22, pp. 5469-5480, 2010.

[31] D. Strumberg, B. Schultheis, U. Traugott et al., "Phase I clinical development of Atu027, a siRNA formulation targeting PKN3 in patients with advanced solid tumors," International Journal of Clinical Pharmacology and Therapeutics, vol. 50, no. 1, pp. 76-78, 2012.

[32] V. Brower, "RNA interference advances to early-stage clinical trials," Journal of the National Cancer Institute, vol. 102, no. 19, pp. 1459-1461, 2010.

[33] D. V. Morrissey, J. A. Lockridge, L. Shaw et al., "Potent and persistent in vivo anti-HBV activity of chemically modified siRNAs," Nature Biotechnology, vol. 23, no. 8, pp. 1002-1007, 2005.

[34] J. Jin, K. H. Bae, H. Yang et al., "In vivo specific delivery of c-Met siRNA to glioblastoma using cationic solid lipid nanoparticles," Bioconjugate Chemistry, vol. 22, no. 12, pp. 2568-2572, 2011.

[35] L. B. Jeffs, L. R. Palmer, E. G. Ambegia, C. Giesbrecht, S. Ewanick, and I. MacLachlan, "A scalable, extrusion-free method for efficient liposomal encapsulation of plasmid DNA," Pharmaceutical Research, vol. 22, no. 3, pp. 362-372, 2005.

[36] T. S. Zimmermann, A. C. H. Lee, A. Akinc et al., "RNAimediated gene silencing in non-human primates," Nature, vol. 441, no. 7089, pp. 111-114, 2006.

[37] A. D. Judge, M. Robbins, I. Tavakoli et al., "Confirming the RNAi-mediated mechanism of action of siRNA-based cancer therapeutics in mice," The Journal of Clinical Investigation, vol. 119, no. 3, pp. 661-673, 2009.

[38] H. Shen, T. Sun, and M. Ferrari, "Nanovector delivery of siRNA for cancer therapy," Cancer Gene Therapy, vol. 19, no. 6, pp. 367373, 2012.

[39] A. Akinc, A. Zumbuehl, M. Goldberg et al., "A combinatorial library of lipid-like materials for delivery of RNAi therapeutics," Nature Biotechnology, vol. 26, no. 5, pp. 561-569, 2008.
[40] A. Akinc, M. Goldberg, J. Qin et al., "Development of lipidoidsirna formulations for systemic delivery to the liver," Molecular Therapy, vol. 17, no. 5, pp. 872-879, 2009.

[41] K. T. Love, K. P. Mahon, C. G. Levins et al., "Lipid-like materials for low-dose, in vivo gene silencing," Proceedings of the National Academy of Sciences of the United States of America, vol. 107, no. 5, pp. 1864-1869, 2010.

[42] S. P. Egusquiaguirre, M. Igartua, R. M. Hernandez, and J. L. Pedraz, "Nanoparticle delivery systems for cancer therapy: advances in clinical and preclinical research," Clinical and Translational Oncology, vol. 14, no. 2, pp. 83-93, 2012.

[43] M. E. Davis and M. E. Brewster, "Cyclodextrin-based pharmaceutics: past, present and future," Nature Reviews Drug Discovery, vol. 3, no. 12, pp. 1023-1035, 2004.

[44] S. Hu-Lieskovan, J. D. Heidel, D. W. Bartlett, M. E. Davis, and T. J. Triche, "Sequence-specific knockdown of EWS-FLI1 by targeted, nonviral delivery of small interfering RNA inhibits tumor growth in a murine model of metastatic Ewing's sarcoma," Cancer Research, vol. 65, no. 19, pp. 8984-8992, 2005.

[45] M. E. Davis, J. E. Zuckerman, C. H. J. Choi et al., "Evidence of RNAi in humans from systemically administered siRNA via targeted nanoparticles," Nature, vol. 464, no. 7291, pp. 10671070, 2010.

[46] K. A. Howard, U. L. Rahbek, X. Liu et al., "RNA interference in vitro and in vivo using a novel chitosan/siRNA nanoparticle system," Molecular Therapy, vol. 14, no. 4, pp. 476-484, 2006.

[47] X. Liu, K. A. Howard, M. Dong et al., "The influence of polymeric properties on chitosan/siRNA nanoparticle formulation and gene silencing," Biomaterials, vol. 28, no. 6, pp. 1280-1288, 2007.

[48] M. Jean, F. Smaoui, M. Lavertu et al., "Chitosan-plasmid nanoparticle formulations for IM and SC delivery of recombinant FGF-2 and PDGF-BB or generation of antibodies," Gene Therapy, vol. 16, no. 9, pp. 1097-1110, 2009.

[49] H. Katas and H. O. Alpar, "Development and characterisation of chitosan nanoparticles for siRNA delivery," Journal of Controlled Release, vol. 115, no. 2, pp. 216-225, 2006.

[50] M. Alameh, D. Dejesus, M. Jean et al., "Low molecular weight chitosan nanoparticulate system at low N:P ratio for nontoxic polynucleotide delivery," International Journal of Nanomedicine, vol. 7, pp. 1399-1414, 2012.

[51] Z. W. Wu, C. T. Chien, C. Y. Liu, J. Y. Yan, and S. Y. Lin, "Recent progress in copolymer-mediated siRNA delivery," Journal of Drug Targeting, vol. 20, no. 7, pp. 551-560, 2012.

[52] O. M. Merkel, A. Beyerle, B. M. Beckmann et al., "Polymerrelated off-target effects in non-viral siRNA delivery," Biomaterials, vol. 32, no. 9, pp. 2388-2398, 2011.

[53] S. M. Moghimi, P. Symonds, J. C. Murray, A. C. Hunter, G. Debska, and A. Szewczyk, "A two-stage poly(ethylenimine)mediated cytotoxicity: implications for gene transfer/therapy," Molecular Therapy, vol. 11, no. 6, pp. 990-995, 2005.

[54] A. C. Hunter and S. M. Moghimi, "Cationic carriers of genetic material and cell death: a mitochondrial tale," Biochimica et Biophysica Acta, vol. 1797, no. 6-7, pp. 1203-1209, 2010.

[55] K. A. Woodrow, Y. Cu, C. J. Booth, J. K. Saucier-Sawyer, M. J. Wood, and W. M. Saltzman, "Intravaginal gene silencing using biodegradable polymer nanoparticles densely loaded with small-interfering RNA," Nature Materials, vol. 8, no. 6, pp. 526533, 2009.

[56] K. Singha, R. Namgung, and W. J. Kim, "Polymers in smallinterfering RNA delivery," Nucleic Acid Therapeutics, vol. 21, no. 3, pp. 133-147, 2011. 
[57] P. Kesharwani, R. K. Tekade, V. Gajbhiye, K. Jain, and N. K. Jain, "Cancer targeting potential of some ligandanchored poly(propylene imine) dendrimers: a comparison," Nanomedicine, vol. 7, no. 3, pp. 295-304, 2011.

[58] J.-H. Lee, K. E. Cha, M. S. Kim et al., "Nanosized polyamidoamine (PAMAM) dendrimer-induced apoptosis mediated by mitochondrial dysfunction," Toxicology Letters, vol. 190, no. 2, pp. 202-207, 2009.

[59] M. L. Patil, M. Zhang, O. Taratula, O. B. Garbuzenko, H. He, and T. Minko, "Internally cationic polyamidoamine PAMAM$\mathrm{OH}$ dendrimers for siRNA delivery: effect of the degree of Quaternization and cancer targeting," Biomacromolecules, vol. 10, no. 2, pp. 258-266, 2009.

[60] O. Taratula, O. B. Garbuzenko, P. Kirkpatrick et al., "Surfaceengineered targeted PPI dendrimer for efficient intracellular and intratumoral siRNA delivery," Journal of Controlled Release, vol. 140, no. 3, pp. 284-293, 2009.

[61] A. Agrawal, D.-H. Min, N. Singh et al., "Functional delivery of siRNA in mice using dendriworms," ACS Nano, vol. 3, no. 9, pp. 2495-2504, 2009.

[62] D. Pantarotto, R. Singh, D. McCarthy et al., "Functionalized carbon nanotubes for plasmid DNA gene delivery," Angewandte Chemie, vol. 43, no. 39, pp. 5242-5246, 2004.

[63] D. Cai, J. M. Mataraza, Z.-H. Qin et al., "Highly efficient molecular delivery into mammalian cells using carbon nanotube spearing," Nature Methods, vol. 2, no. 6, pp. 449-454, 2005.

[64] P. Kesharwani, V. Gajbhiye, and N. K. Jain, "A review of nanocarriers for the delivery of small interfering RNA," Biomaterials, vol. 33, no. 29, pp. 7138-7150, 2012.

[65] Z. Zhang, X. Yang, Y. Zhang et al., "Delivery of telomerase reverse transcriptase small interfering RNA in complex with positively charged single-walled carbon nanotubes suppresses tumor growth," Clinical Cancer Research, vol. 12, no. 16, pp. 4933-4939, 2006.

[66] Z. Liu, M. Winters, M. Holodniy, and H. Dai, "siRNA delivery into human $\mathrm{T}$ cells and primary cells with carbon-nanotube transporters," Angewandte Chemie, vol. 46, no. 12, pp. 20232027, 2007.

[67] H. Wang, J. Wang, X. Deng et al., "Biodistribution of carbon single-wall carbon nanotubes in mice," Journal of Nanoscience and Nanotechnology, vol. 4, no. 8, pp. 1019-1024, 2004.

[68] K. T. Al-Jamal, F. M. Toma, A. Yilmazer et al., "Enhanced cellular internalization and gene silencing with a series of cationic dendron-multiwalled carbon nanotube:siRNA complexes," FASEB Journal, vol. 24, no. 11, pp. 4354-4365, 2010.

[69] J. E. Podesta, K. T. Al-Jamal, M. A. Herrero et al., "Antitumor activity and prolonged survival by carbon-nanotube-mediated therapeutic sirna silencing in a human lung xenograft model," Small, vol. 5, no. 10, pp. 1176-1185, 2009.

[70] A. A. Shvedova, E. R. Kisin, D. Porter et al., "Mechanisms of pulmonary toxicity and medical applications of carbon nanotubes: two faces of Janus?" Pharmacology \& Therapeutics, vol. 121, no. 2, pp. 192-204, 2009.

[71] C. P. Firme III and P. R. Bandaru, "Toxicity issues in the application of carbon nanotubes to biological systems," Nanomedicine, vol. 6, no. 2, pp. 245-256, 2010.

[72] Y. Yu and D. Sun, "Superparamagnetic iron oxide nanoparticle 'theranostics' for multimodality tumor imaging, gene delivery, targeted drug and prodrug delivery," Expert Review of Clinical Pharmacology, vol. 3, no. 1, pp. 117-130, 2010.
[73] X.-H. Peng, X. Qian, H. Mao et al., "Targeted magnetic iron oxide nanoparticles for tumor imaging and therapy," International Journal of Nanomedicine, vol. 3, no. 3, pp. 311-321, 2008.

[74] Z. Medarova, W. Pham, C. Farrar, V. Petkova, and A. Moore, "In vivo imaging of siRNA delivery and silencing in tumors," Nature Medicine, vol. 13, no. 3, pp. 372-377, 2007.

[75] J.-H. Lee, K. Lee, S. H. Moon, Y. Lee, T. G. Park, and J. Cheon, "All-in-one target-cell-specific magnetic nanoparticles for simultaneous molecular imaging and siRNA delivery," Angewandte Chemie, vol. 48, no. 23, pp. 4174-4179, 2009.

[76] Y.-S. Cho, T.-J. Yoon, E.-S. Jang et al., "Cetuximab-conjugated magneto-fluorescent silica nanoparticles for in vivo colon cancer targeting and imaging," Cancer Letters, vol. 299, no. 1, pp. 63-71, 2010.

[77] A. M. Smith, H. Duan, A. M. Mohs, and S. Nie, "Bioconjugated quantum dots for in vivo molecular and cellular imaging," Advanced Drug Delivery Reviews, vol. 60, no. 11, pp. 1226-1240, 2008.

[78] A. M. Derfus, A. A. Chen, D.-H. Min, E. Ruoslahti, and S. N. Bhatia, "Targeted quantum dot conjugates for siRNA delivery," Bioconjugate Chemistry, vol. 18, no. 5, pp. 1391-1396, 2007.

[79] W. B. Tan, S. Jiang, and Y. Zhang, "Quantum-dot based nanoparticles for targeted silencing of HER $2 /$ neu gene via RNA interference," Biomaterials, vol. 28, no. 8, pp. 1565-1571, 2007.

[80] M. V. Yezhelyev, L. Qi, R. M. O'Regan, S. Nie, and X. Gao, "Proton-sponge coated quantum dots for siRNA delivery and intracellular imaging," Journal of the American Chemical Society, vol. 130, no. 28, pp. 9006-9012, 2008.

[81] Y. Su, Y. He, H. Lu et al., “The cytotoxicity of cadmium based, aqueous phase-synthesized, quantum dots and its modulation by surface coating," Biomaterials, vol. 30, no. 1, pp. 19-25, 2009.

[82] P. Subramaniam, S. J. Lee, S. Shah, S. Patel, V. Starovoytov, and K. B. Lee, "Generation of a library of non-toxic quantum dots for cellular imaging and siRNA delivery," Advanced Materials, vol. 24, no. 29, pp. 4014-4019, 2012.

[83] P. Ghosh, G. Han, M. De, C. K. Kim, and V. M. Rotello, "Gold nanoparticles in delivery applications," Advanced Drug Delivery Reviews, vol. 60, no. 11, pp. 1307-1315, 2008.

[84] N. L. Rosi, D. A. Giljohann, C. S. Thaxton, A. K. R. Lytton-Jean, M. S. Han, and C. A. Mirkin, "Oligonucleotide-modified gold nanoparticles for infracellular gene regulation," Science, vol. 312, no. 5776, pp. 1027-1030, 2006.

[85] W. H. Kong, K. H. Bae, S. D. Jo, J. S. Kim, and T. G. Park, "Cationic lipid-coated gold nanoparticles as efficient and noncytotoxic intracellular siRNA delivery vehicles," Pharmaceutical Research, vol. 29, no. 2, pp. 362-374, 2012.

[86] A. C. Bonoiu, S. D. Mahajan, H. Ding et al., "Nanotechnology approach for drug addiction therapy: gene silencing using delivery of gold nanorod-siRNA nanoplex in dopaminergic neurons," Proceedings of the National Academy of Sciences of the United States of America, vol. 106, no. 14, pp. 5546-5550, 2009.

[87] E.-Y. Kim, R. Schulz, P. Swantek, K. Kunstman, M. H. Malim, and S. M. Wolinsky, "Gold nanoparticle-mediated gene delivery induces widespread changes in the expression of innate immunity genes," Gene Therapy, vol. 19, no. 3, pp. 347-353, 2012.

[88] E. Song, P. Zhu, S.-K. Lee et al., "Antibody mediated in vivo delivery of small interfering RNAs via cell-surface receptors," Nature Biotechnology, vol. 23, no. 6, pp. 709-717, 2005.

[89] P. Kumar, H.-S. Ban, S.-S. Kim et al., "T cell-specific siRNA delivery suppresses HIV-1 infection in humanized mice," Cell, vol. 134, no. 4, pp. 577-586, 2008. 
[90] J. O. McNamara II, E. R. Andrechek, Y. Wang et al., "Cell typespecific delivery of siRNAs with aptamer-siRNA chimeras," Nature Biotechnology, vol. 24, no. 8, pp. 1005-1015, 2006.

[91] P. Guo, O. Coban, N. M. Snead et al., "Engineering rna for targeted sirna delivery and medical application," Advanced Drug Delivery Reviews, vol. 62, no. 6, pp. 650-666, 2010.

[92] S. Abdelmawla, S. Guo, L. Zhang et al., "Pharmacological characterization of chemically synthesized monomeric phi29 pRNA nanoparticles for systemic delivery," Molecular Therapy, vol. 19, no. 7, pp. 1312-1322, 2011.

[93] D. Shu, Y. Shu, F. Haque, S. Abdelmawla, and P. Guo, “Thermodynamically stable RNA three-way junction for constructing multifunctional nanoparticles for delivery of therapeutics," Nature Nanotechnology, vol. 6, no. 10, pp. 658-667, 2011.

[94] F. Haque, D. Shu, Y. Shu et al., "Ultrastable synergistic tetravalent RNA nanoparticles for targeting to cancers," Nano Today, vol. 7, no. 4, pp. 245-257, 2012.

[95] B. R. Meade and S. F. Dowdy, "Exogenous siRNA delivery using peptide transduction domains/cell penetrating peptides," Advanced Drug Delivery Reviews, vol. 59, no. 2-3, pp. 134-140, 2007.

[96] S. A. Moschos, S. W. Jones, M. M. Perry et al., "Lung delivery studies using siRNA conjugated to TAT(48-60) and penetratin reveal peptide induced reduction in gene expression and induction of innate immunity," Bioconjugate Chemistry, vol. 18, no. 5, pp. 1450-1459, 2007.

[97] Y. S. Cho, G. Y. Lee, H. K. Sajja et al., "Targeted delivery of siRNA-generating DNA nanocassettes using multifunctional nanoparticles," Small, 2013.

[98] B. S. Nielsen, F. Rank, M. Illemann, L. R. Lund, and K. Danø, "Stromal cells associated with early invasive foci in human mammary ductal carcinoma in situ coexpress urokinase and urokinase receptor," International Journal of Cancer, vol. 120, no. 10, pp. 2086-2095, 2007.

[99] F. Blasi and P. Carmeliet, "uPAR: a versatile signalling orchestrator," Nature Reviews Molecular Cell Biology, vol. 3, no. 12, pp. 932-943, 2002.

[100] A. L. Jackson, S. R. Bartz, J. Schelter et al., "Expression profiling reveals off-target gene regulation by RNAi," Nature Biotechnology, vol. 21, no. 6, pp. 635-637, 2003. 

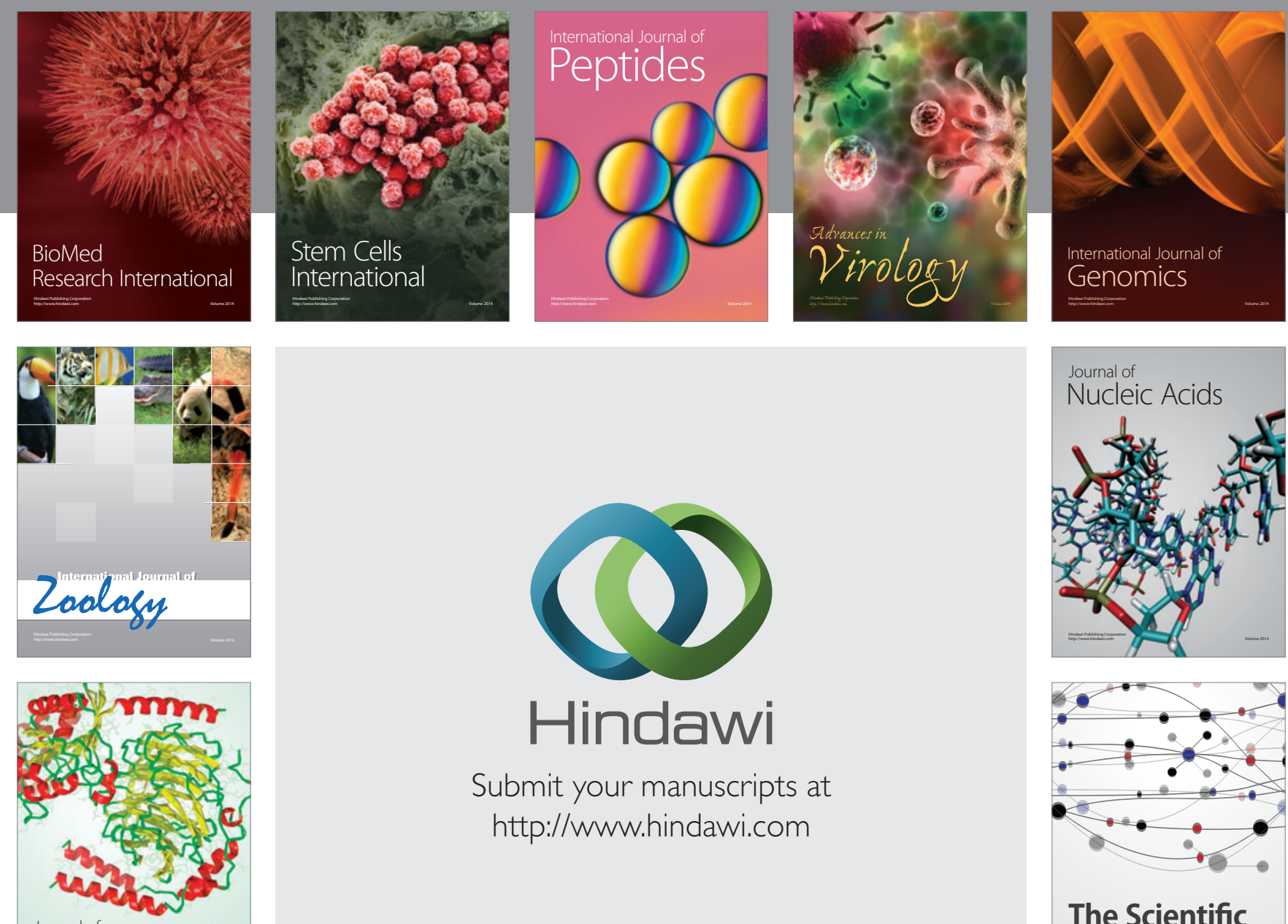

Submit your manuscripts at

http://www.hindawi.com

Journal of
Signal Transduction
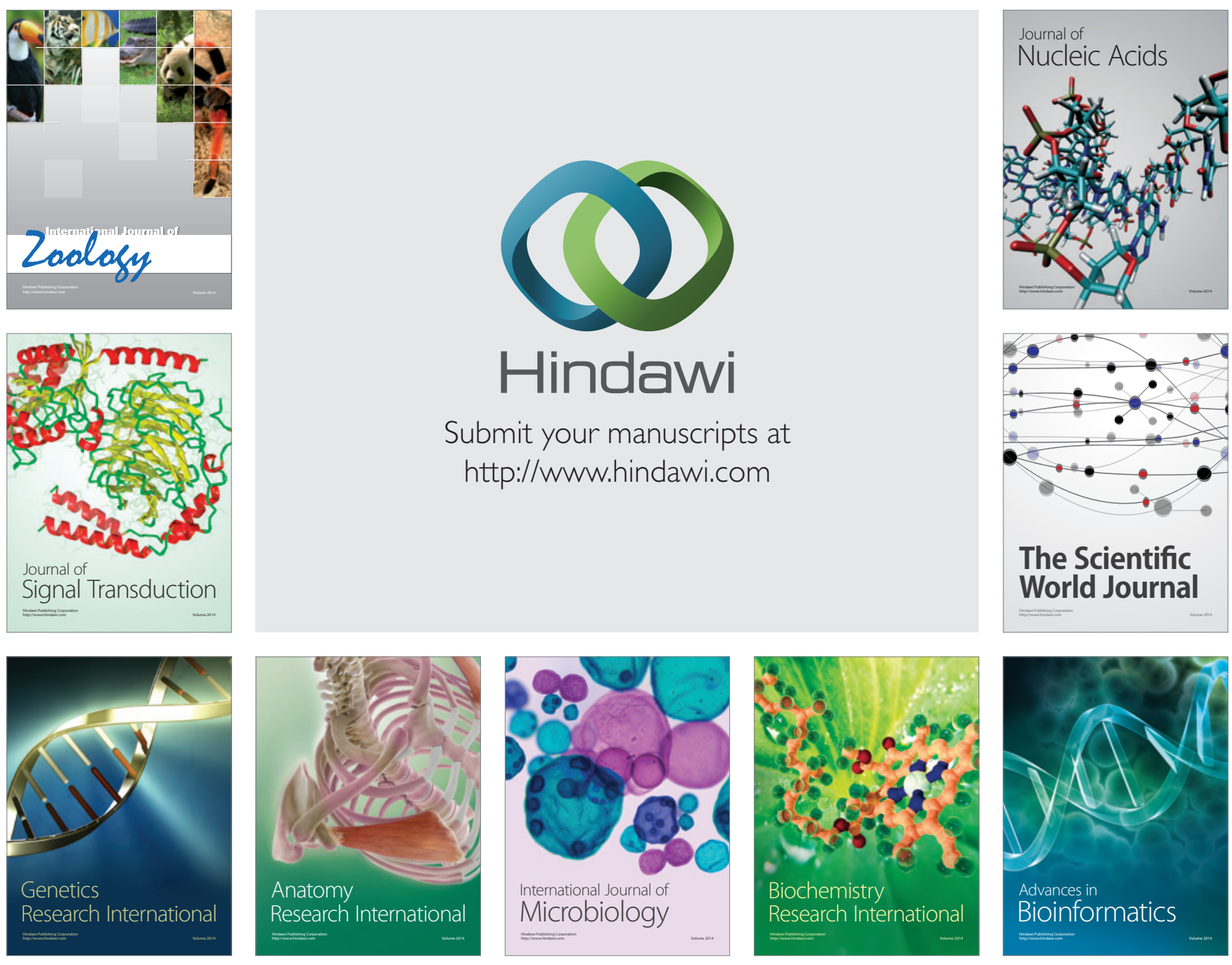

The Scientific World Journal
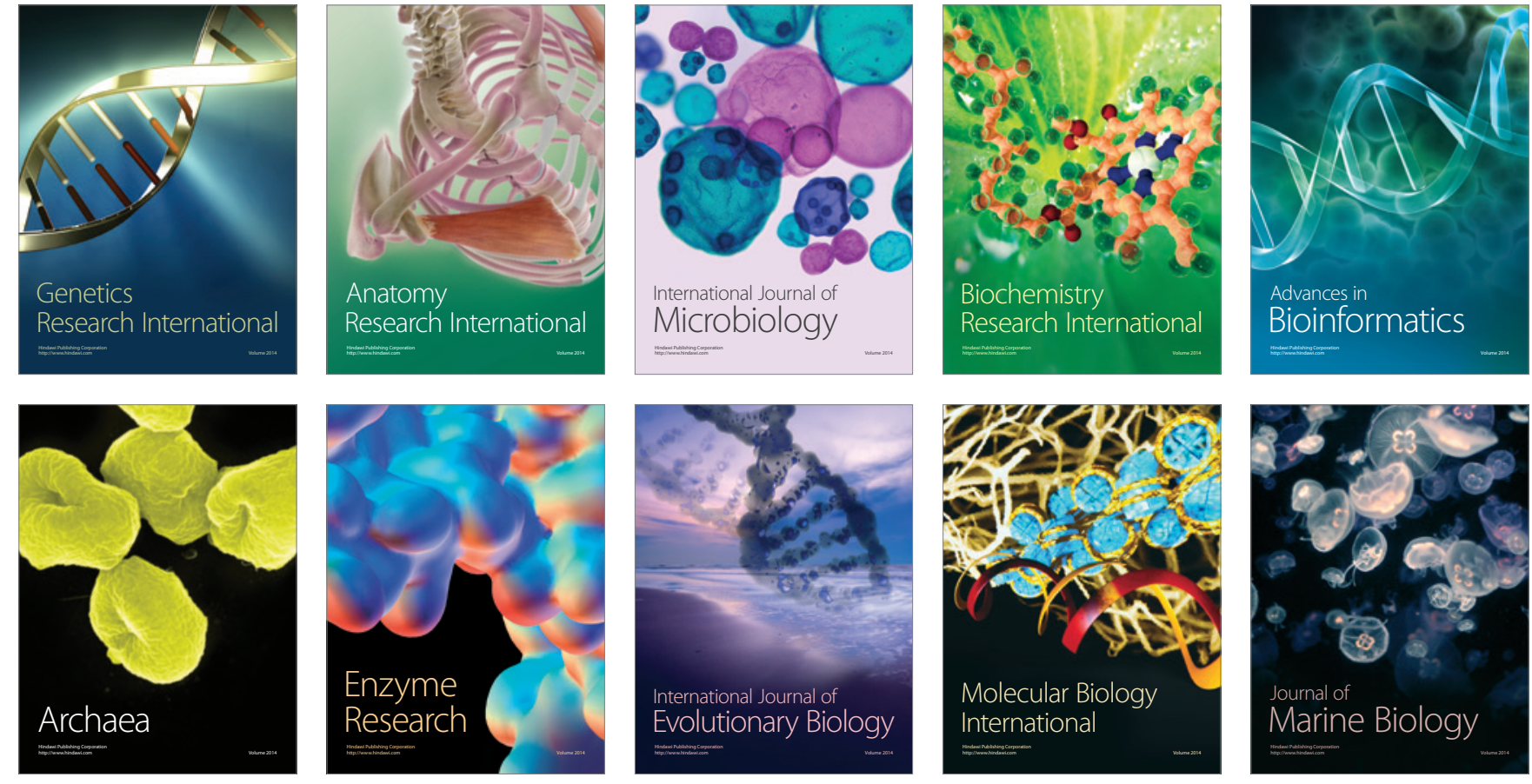